\title{
Deadbeat State Space Control of Spinning Satellite
}

\author{
Ezzeddin M. Elarbi ${ }^{1}$ and Saad M. Issa ${ }^{2}$
}

Accepted $15^{\text {th }}$ August 2014

DOI: 10.18100/ijamec.21419

\begin{abstract}
The colony of a celestial universe has become foremost task for many countries in the late decades. The spinning attitude control for economical satellite operation is widely used. This paper shows a successful implementation of state space technique to inherently unstable satellite spun around longitudinal axis. Full-state feedback and reference input with full-state feedback are compared for deadbeat response. The state space control gains are tuned to satisfy controllability conditions. Observability conditions are checked for better tracking of attitude spinning angle to step input with the aim to achieve a robust spin stabilization of the satellite manoeuvre. Satisfactory deadbeat specifications are obtained based on reference input with full-state feedback. However, full-state feedback implements reasonable design gains and it appears to be slower in judgment with reference input with full-state feedback gains. The simulation and analytical results are well agreed based on dominant second-order poles.
\end{abstract}

Keywords: Spinning Satellite Stabilization, Deadbeat Control, State Space, Pole Placement Method, FSF and RIFSF controllers.

Nomenclatures

$$
\begin{aligned}
& T_{x}, T_{y}, T_{z} \\
& \text { Torques around } x, y \text { and } z \text { axes }[\mathrm{N} \mathrm{m}] \text {. } \\
& I_{x}, I_{y}, I_{z} \\
& K=\left[\begin{array}{ll}
K_{1} & K_{2}
\end{array}\right] \\
& \text { Mass moment of intertie around } x, y \text { and } z \\
& \text { axes }\left[\mathrm{Kg} \mathrm{m}^{2}\right] \text {. } \\
& \alpha_{x}, \alpha_{y}, \alpha_{z} \\
& \text { Full state feedback vector } \\
& \text { Angular accelerations Around } x, y \text { and } z \\
& \text { axes }\left[\mathrm{rad} / \mathrm{sec}^{2}\right] \text {. } \\
& \bar{N} \quad \text { Overall reference input gain } \\
& \left.\omega_{n} \quad \text { Undamped frequency [ } \mathrm{rad} / \mathrm{sec}\right] \text {. } \\
& N_{u} \quad \text { Explicit reference input gain } \\
& \omega_{x}, \omega_{y}, \omega_{z} \quad \text { Angular velocities around } x, y \text { and } z \text { axes } \\
& {[\mathrm{rad} / \mathrm{sec}] \text {. }} \\
& N_{x}=\left[\begin{array}{ll}
N_{x 1} & N_{x 2}
\end{array}\right] \text { Reference input gains corresponds with } \\
& \text { full state feedback vector }
\end{aligned}
$$

\section{Introduction}

The adventure into space is usually undertaken for scientific missions, communications, surveillances and reconnaissance. The pioneering space explorations are made by the soviet union and united states at the late of 1950s [1]. By the end of past century it has seen partnerships many countries such as Japan and Europe to invade the space. Lastly, Chine and India ride the race into space due to the availability of various resources. Spinning attitude control is one of strategies for long life mission for more than 40 years. Propulsion system thrusters are fired only occasionally to make desired changes in the spinstabilized attitude. At the present time, most of the satellite attitude controls in practical world are based on a proportionalintegral-derivative (Pid) controller algorithm [2]. The state space $(S S)$ method is a simple technique to describe satellite

\footnotetext{
${ }^{1,2}$ Faculty of Engineering, University of Tripoli, Libya

* Corresponding Author: Email: sissa@aerodept.edu.ly

\# This paper has been presented at the International Conference on

Advanced Technology\&Sciences (ICAT'14) held in Antalya (Turkey), August 12-15, 2014.
}

spun around longitudinal axis. The SS method has been in a use for over 100 years and is introduced to control designs in the ate 1950 s by R. E. Kalman [3].

Beginning in the late 1950 s and early 1960s Kalman introduced the ideas of state-variables, controllability and observability. Zadeh and Desoer had a significant impact in promoting the state-space method. During the 1980s reliable numerical methods were developed for dealing with statevariable designs and computer-aided software for control design [4]. Since the invention of Matlab by Cleve Miler huge applications for control design were made. The state-variable methods were gaining momentum particularly in the US. Research groups in England led by Rosenbrock, MacFarlane, and Munro extended to multi-input multi-output systems. In the end of the 1980s the frequency domain method is used in connection with state-variable method as powerful approach [5]. The state-variable methods were found more computationally advantageous than the frequency domain method [6].

This paper is devoted to implement $S S$ controller algorithms to unstable spinning satellite. The performance of Reference Input with Full State Feedback (RIFSF) and Full State Feedback $(F S F)$ controller are examined for deadbeat specifications. A Matlab framework of M-file batch and Simulink is used to model, simulate and control spinning satellite.

\section{Mathematical model}

The brief demonstrations of satellite problem descriptions, control algorithms and implementations will be described below. Figure.1 illustrates the diagram of a spinning satellite with SS controller implementation. The operator is just MatlabM-file script used to command Matlab-Simulink to perform the simulation tasks and return back the results for post processing. The SS controller block is used to implement one time RIFSF and another time FSF controller. 


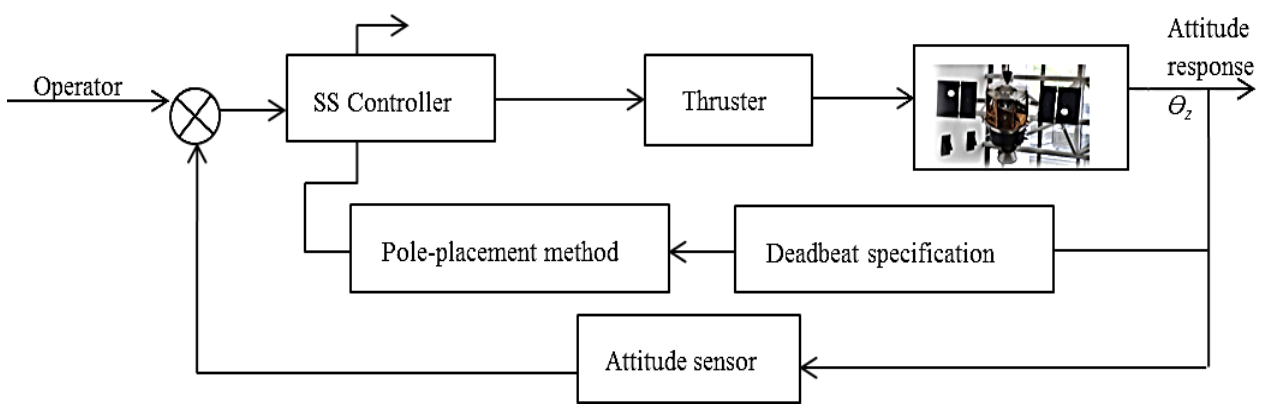

A. Figure 1. A schematic of spinning satellite control based on SS algorithm

\section{A. Satellite Dynamic Model:}

The satellite under consideration is an axis-symmetric rigid satellite of evenly distributed masses and space disturbance free. Of particular intention, the satellite is considered inherently unstable due to two poles at the origin. The satellite moment of inertia matrix is given by [7].

$$
\left[\begin{array}{ccc}
I_{x} & I_{x y} & I_{x z} \\
I_{x y} & I_{y} & I_{y z} \\
I_{x z} & I_{y z} & I_{z}
\end{array}\right]=\left[\begin{array}{ccc}
1000 & 0 & 0 \\
0 & 1000 & 0 \\
0 & 0 & 1000
\end{array}\right] \mathrm{kgm}^{2}
$$

The satellite cannot keep its balance status and it could not come back to its initial orientation until controller is implemented. The non-linear equations of satellite motion can be shown as [8].

$$
\begin{aligned}
& T_{x}=I_{x} \alpha_{x}-\left(I_{y}-I_{z}\right) \omega_{y} \omega_{z} \\
& T_{y}=I_{y} \alpha_{y}-\left(I_{z}-I_{x}\right) \omega_{z} \omega_{x} \\
& T_{z}=I_{z} \alpha_{z}-\left(I_{x}-I_{y}\right) \omega_{x} \omega_{y}
\end{aligned}
$$

Highly nonlinear characteristics of satellite dynamic system are linearized in a form of double-integral plant. There exists a linearized set of equation of motion, the coupling terms of angular velocities may be neglected for a small roll, pitch and yaw attitudes [9].

$$
\begin{aligned}
& T_{x}=I_{x} \alpha_{x} \\
& T_{y}=I_{y} \alpha_{y} \\
& T_{z}=I_{z} \alpha_{z}
\end{aligned}
$$

To model the satellite spinning around the longitude axis where the moment of inertia is the largest, equation (2) will be modelled as

$$
T_{z}=I_{z} \alpha_{z}=I_{z} \dot{\omega}_{z}=I_{z} \ddot{\theta}_{z}
$$

Because the system is linear, the step response can be derived for any input amplitude. The state-variable method is used to describe the satellite dynamics. A set of first-order differential equations in the vector-valued state of the system is composed below

$$
\begin{aligned}
& x_{1}=\theta_{z} \\
& \dot{x}_{1}=x_{2}=\dot{\theta}_{z}=\omega_{z} \\
& \dot{x}_{2}=\ddot{\theta}_{z}=\alpha_{z}=\frac{T_{z}}{I_{z}}+M_{d}
\end{aligned}
$$

Where $\boldsymbol{x}_{\mathbf{1}}$ and $\boldsymbol{x}_{\mathbf{2}}$ are state variables, and $\boldsymbol{\theta}_{z}$ is the spinning angular displacement around $\mathrm{z}$ axis.

The SS form can be given by

$$
\left.\begin{array}{c}
\dot{x}=A x+B u \\
\theta_{z}=C x+D u
\end{array}\right\}
$$

Where $u$ is control effort and $A$ is system matrix, $B$ is input matrix, $C$ is output matrix and $\mathrm{D}$ is direct transmission matrix, which are:

$$
A=\left[\begin{array}{ll}
0 & 1 \\
0 & 0
\end{array}\right], B=\left[\begin{array}{ll}
0 & \frac{1}{I_{z z}}
\end{array}\right]^{T}, C=\left[\begin{array}{ll}
1 & 0
\end{array}\right] \text { and } D=[0]
$$

In this study external disturbance $\left(\boldsymbol{M}_{\boldsymbol{d}}\right)$ represented the solar pressure is not considered.

\section{B. Control Law Algorithms:}

Two concepts of control design working in connection with SS method are tested. FSF and RIFSF are applied and compared to assign a set of pole locations for the closed-loop system that is satisfactory dynamic transient response.

$$
u=-K x=-\left[\begin{array}{llll}
K_{1} & K_{2} & \ldots & K_{n}
\end{array}\right]\left[\begin{array}{c}
x_{1} \\
x_{2} \\
\vdots \\
x_{n}
\end{array}\right]
$$

The transfer function of spinning satellite implementing FSF controller can be expressed as

$$
\frac{x_{1}}{r}=\frac{\theta_{z}}{r} \frac{1}{I_{z} s^{2}+K_{2} s+K_{1}}
$$

Obviously, FSF implementation has two poles which are directly influenced by the satellite design, i.e., the moment of inertia and control gains.

\section{2) RIFSF :}

For zero steady state error to step input, introducing the reference input into the system results in changing the control law as follows

$$
\begin{gathered}
u=-K x+\bar{N} r=-\left[\begin{array}{llll}
K_{1} & K_{2} & \ldots & K_{n}
\end{array}\right]\left[\begin{array}{c}
x_{1} \\
x_{2} \\
\vdots \\
x_{n}
\end{array}\right]+\bar{N} r \\
\bar{N}=N_{u}+K N_{x}
\end{gathered}
$$




$$
\left[\begin{array}{l}
N_{x} \\
N_{u}
\end{array}\right]=\left[\begin{array}{ll}
A & B \\
C & D
\end{array}\right]^{-1}\left[\begin{array}{l}
0 \\
1
\end{array}\right]
$$

The transfer function of spinning satellite implementing RIFSF controller can be expressed as

$$
\frac{x_{2}}{r}=\frac{\theta_{z}}{r} \frac{N_{x 1} K_{1}+N_{x 2} K_{2}}{I_{z} s^{2}+K_{2} s+K_{1}}
$$

Again, RIFSF implementation has two poles which are directly influenced by the satellite design, i.e., the moment of inertia and control gains. However, the reference input constants have an implicit effect on the system performance as they appear as gain in the numerator.

\section{Pole Placement Method}

Using Eq.(6) the Eq.(5) becomes

$$
\dot{x}=A x-B K x
$$

The characteristic equation of the closed-loop system is

$$
\operatorname{det}[s I-(A-B K)]
$$

$\mathrm{An} \mathrm{n}^{\text {th }}$ order polynomial in s containing the gains $\mathrm{K} 1, \ldots, \mathrm{Kn}$. Assuming the desired pole roots locations are

$$
s=s_{1}, s_{2}, \ldots, s_{n}
$$

The corresponding control characteristic equation is

$$
\left(S-S_{1}\right)\left(S-S_{2}\right) . .\left(S-S_{n}\right)=0
$$

The $K$ vector is obtained by matching coefficients of Eq.(15) with the characteristic equations of Eqs.(7) and (11).

The dominant second order poles approach is considered here due to feasibility when higher order characteristic equations. In this method the poles are selected to satisfy the two conditions of controllability and observability.

The controllability matrix $S$ is computed as

$$
\varsigma=\left[\begin{array}{llll}
B & A B & \ldots & B A^{n-1}
\end{array}\right]^{T}
$$

The system is said to be controllable for controllability matrix $\varsigma$ is non-singular.

The observability matrix $\mathrm{O}$ is calculated by

$$
O=\left[\begin{array}{llll}
C & C A & \ldots & C A^{n-1}
\end{array}\right]^{T}
$$

The system is said to be observable for the observability matrix $O$ is non-singular.

\section{Model and Simulation}

The implementations of FSF and RIFSF controller into spinning satellite dynamic model are schematically shown in (Figures. 2 \& 3). A Matlab M-file script was used to provide workability to simulation model. The results obtained from the simulations are returned back to the script to post process them. The uncontrolled satellite has transfer function

$$
\text { Uncontrolled satellite }: \frac{x_{1}}{r}=\frac{\theta_{z}}{r}=\frac{1}{5000 s^{2}}
$$

Where $r$ is reference input command and the overall transfer functions of FSF and RIFSF controllers for a spinning satellite is given respectively.

$$
\text { FSF comtroller }: \frac{x_{1}}{r}=\frac{\theta_{z}}{r}=\frac{1}{5000 s^{2}+125 s+1}
$$
and

RIFSF comtroller $: \frac{x_{1}}{r}=\frac{\theta_{z}}{r}=\frac{80000}{5000 s^{2}+36000 s+80000}$

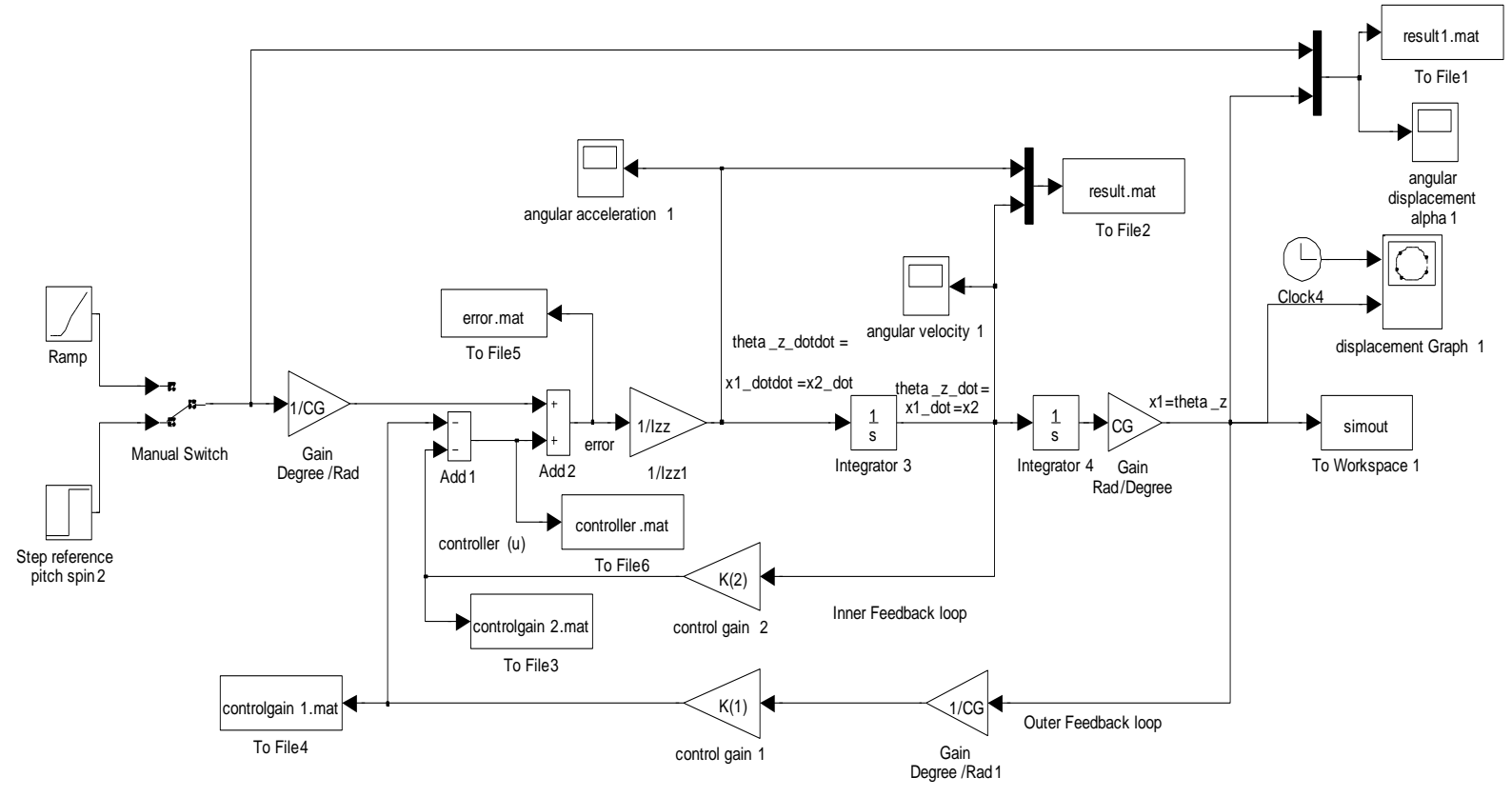

Figure 2. A simulation diagram of $F S F$ controller of spinning satellite 


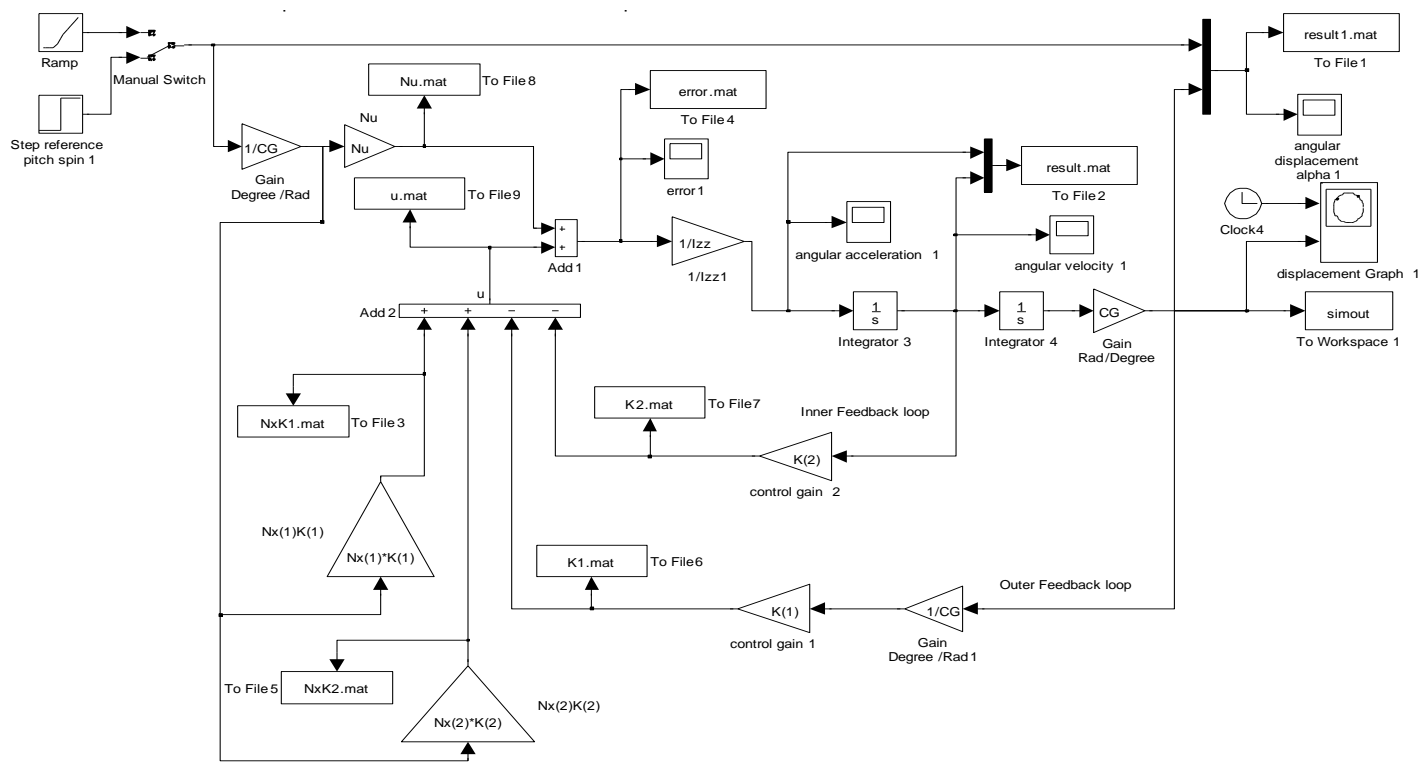

Figure 3. A simulation diagram of RIFSF controller of spinning satellite

\section{Results and Discussions}

The main results obtained in this study, are shown and a brief discussions are outline to elucidate the implication of the results.

\section{E. System Eigenvalues}

Figure.4 shows the root locus comparisons before implementing control algorithms with FSF and RIFSF controllers. Two poles at the origin of uncontrolled satellite make the spinning unstable. Stable eigenvalues of $-3.6000 \pm$ $1.7436 \mathrm{i}$ and $-0.0125 \pm 0.0066 \mathrm{i}$ are produced by RIFSF and FSF controllers respectively. For compact root locus comparisons with uncontrolled satellite and FSF controller, RIFSF gains are normalised by 100 . Cleary, just the real part of eigenvalues of RIFSF controller is normalised by 100 but the imaginary part is normalised by about 4.5. The locations of eigenvalues of uncontrolled satellite are moved by RIFSF and FSF controllers to the left hand side of s-plane but they are not too far from the original locations based on FSF controller. Evidently, RIFSF controller has more stable roots than FSF controller since the eigenvalues are far away in the left hand side of s-plane. Figure. 5 shows a close up of root locus of uncontrolled satellite and FSF controllers. Although the real part of FSF eigenvalues are too close to the poles of uncontrolled satellite the imaginary part of FSF eigenvalues produces damped oscillation to unstable response of spinning satellite.

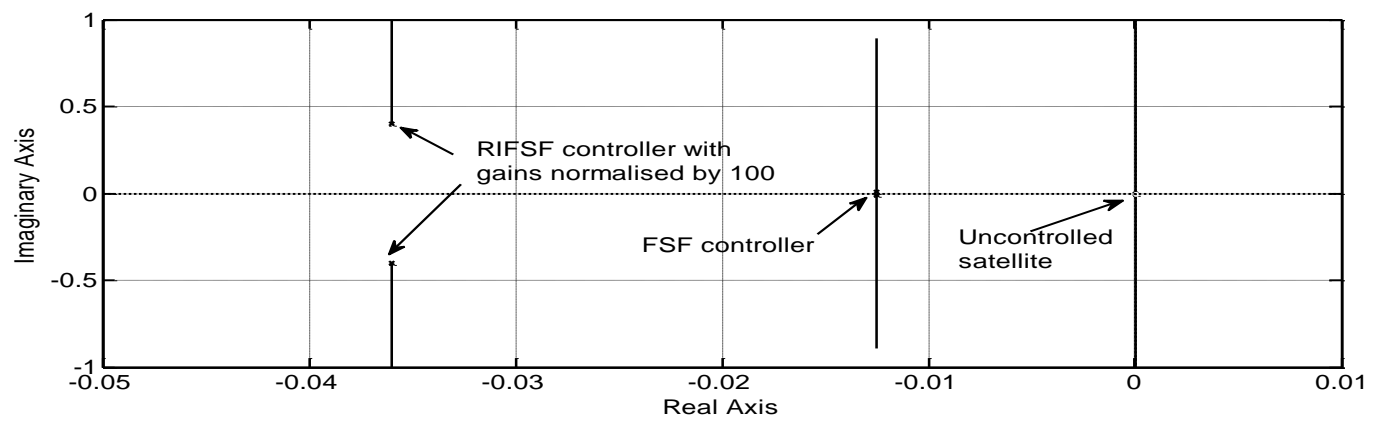

Figure 4. Root locus of uncontrolled satellite with RIFSF and FSF controllers

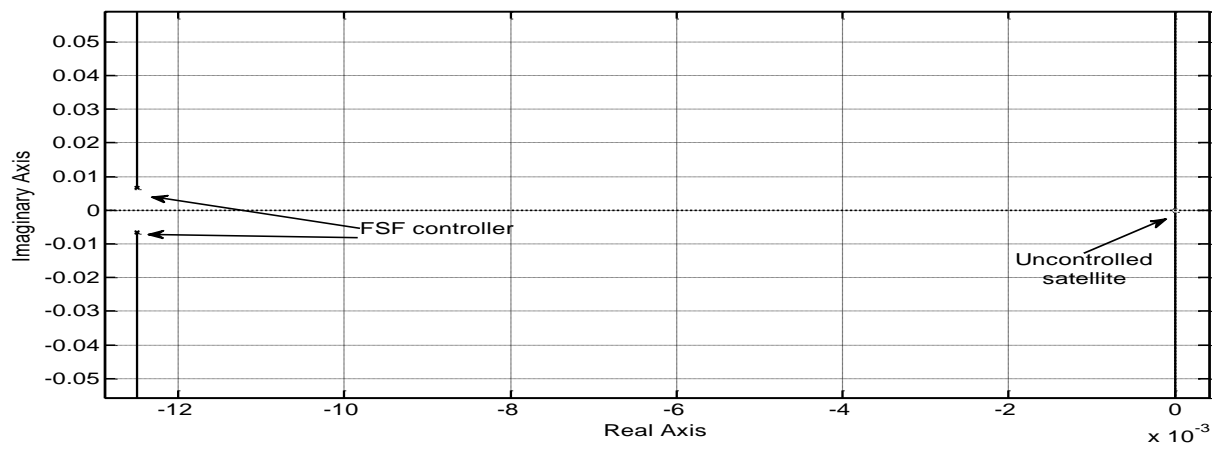

Figure 5. A close up view of root locus of uncontrolled satellite and FSF controller 


\section{F. Controller Gain Contribution}

The contributions of each part of controller effort $u\left(N_{x 1} K_{1}\right.$, $N_{x 2} K_{2}, N_{u}, K_{1} \theta_{z}$ and $\left.K_{2} \theta_{z}\right)$ is investigated based on both RIFSF and FSF control implementations to uncontrolled spinning satellite. Figure.6 shows the contributions of FSF controller gains whereas (Figure.7) displays the contributions of RIFSF controller gains. The convergence of FSF control effort u occurs too late at about $400 \mathrm{sec}$, shown by starred line. The contribution of $\boldsymbol{K}_{\mathbf{2}} \dot{\boldsymbol{\theta}}_{z}$ part (diamond line) has the averaged value of 0.0175 which is eliminated by -0.0175 produced with
$K_{1} \theta_{z}$ part (squared line). On the contrary, a fast convergence is obtained from RIFSF control effort $u$ at an early time. Clearly, a fluctuating convergence around zero is seen by a starred line in (Figure.8) and the most contribution to u comes from $\boldsymbol{K}_{\mathbf{2}} \dot{\boldsymbol{\theta}}_{z}$ part (diamond line). However, the contributions of $\boldsymbol{N}_{\boldsymbol{u}}$ (triangular mark) and $\boldsymbol{N}_{\boldsymbol{x} 2} \boldsymbol{K}_{\mathbf{2}}$ (plus mark) are considerably small. Nevertheless, the quite large contribution of $N_{x 1} K_{1}$ (circled line) is cancelled with $K_{1} \theta_{z}$ part (squared line).

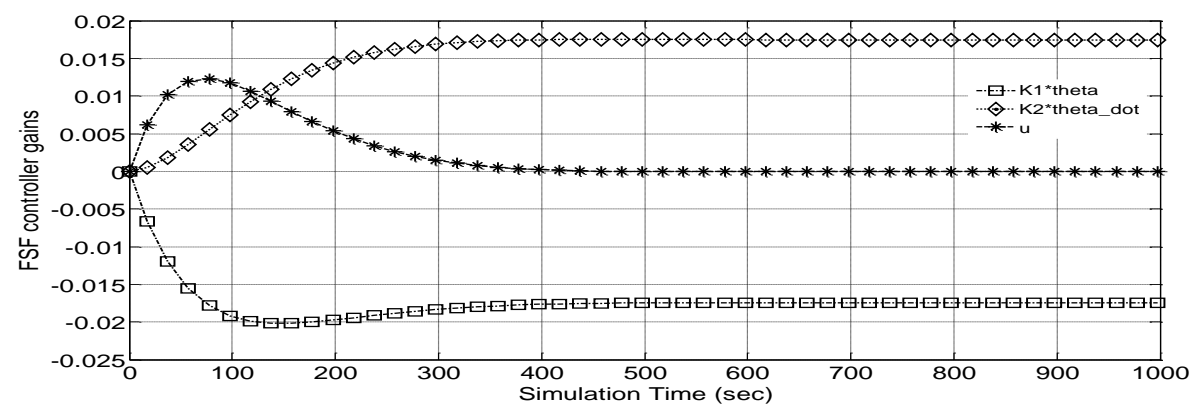

Fig. 6 The contributions of $F S F$ controller gains

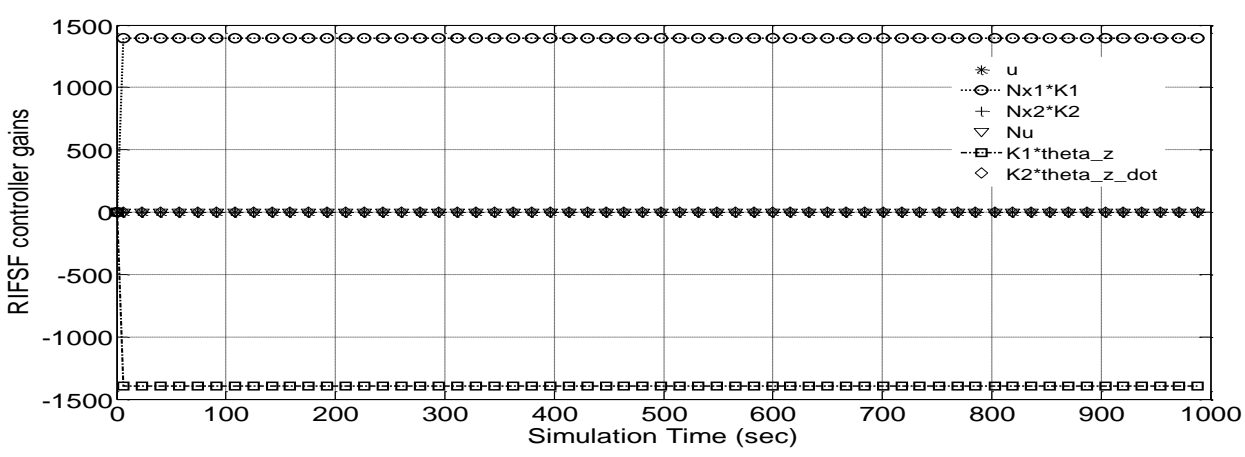

Figure 7. The contributions of RIFSF controller gains

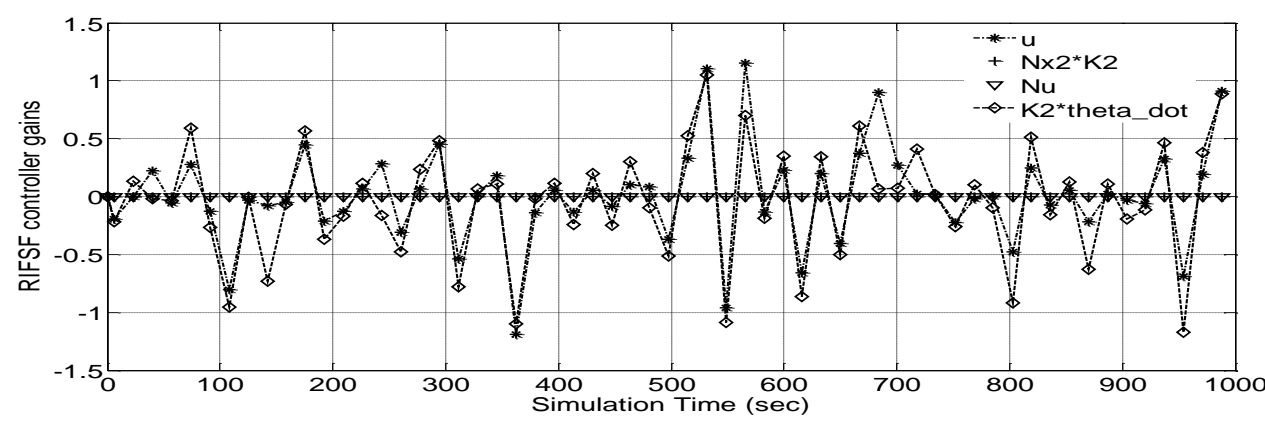

Figure 8. A close up view of the contributions of RIFSF controller gains

\section{G. Deadbeat Response}

A comparison of the performance of FSF and RIFSF controllers for a spinning satellite is shown in (Figure.9). Clearly, RIFSF performs well to meet the deadbeat specifications. Although RIFSF controller adequately tracks unity step input command it implements expansive impractical gains. However, FSF controller is not too bad for practical implementations since it deploys reasonable gains. A close up view of the response obtained by RIFSF and FSF controllers during the simulation time between $0-450 \mathrm{sec}$ is shown in (Figure.10). Obviously, FSF is slower than RIFSF in terms of rise and settling times. Figure. 11 shows a comparison of steady state convergence between FSF and RIFSF controllers. Apparently, RIFSF controller promptly shows steady state behaviour in a comparison with FSF controller which converges at approximately $800 \mathrm{sec}$. An average of zero steady state error is approximately produced by RIFSF controller in contrast to $F S F$ controller which produces about $0.4 \%$ steady state error. The smooth convergence which is seen from FSF controller implementation refers to the contribution seen by the control effort $\mathrm{u}$ shown in (Figure.8). However, the fluctuating convergence based on RIFSF controller implementation (Figure. 11) is due to that was produced by the control effort $\mathrm{u}$ as seen in (Figure. 8). 
The simulation and analytical results are agreed well based on dominant second-order poles. As early shown, RIFSF and $F S F$ have dominate poles at $-3.6000 \pm 1.7436 \mathrm{i}$ and $-0.0125 \pm$ $0.0066 \mathrm{i}$ respectively. Standard performance measures are usually defined in terms of the step response. The swiftness of response is measured by the peak time $\boldsymbol{T}_{\boldsymbol{p}}$ and rise time $\boldsymbol{T}_{\boldsymbol{r}}$. However, the track to desired response is examined by the settling time $\boldsymbol{T}_{s}$ and the percent overshoot P.O. The peak time TP is calculated by

$$
T_{p}=\frac{\pi}{\omega_{n} \sqrt{1-\zeta^{2}}}
$$

The liner approximation of analytical expression of rise time $\mathrm{Tr}$ can be found by

$$
T_{r}=\frac{2.16 \zeta+0.6}{\omega_{n}}
$$

The settling time TS for which the response remains within $2 \%$ of the final values is given by

$$
T_{s}=\frac{4}{\zeta \omega_{n}}
$$

The present overshoot is found by

$$
\text { P.O. }=100 e^{-\zeta \pi / \sqrt{1-\zeta^{2}}}
$$

Table (1) shows a comparison between the simulation results obtained from RIFSF controller for spinning stabilisation satellite and analytical calculations based on Eqs. (18-21). Table (2) shows a comparison between the simulation results obtained from FSF controller for spinning stabilisation satellite and analytical calculations based on Eqs. (18-21). FSF controller has the damping ratio $\zeta$ of 0.885 and the undamped frequency $\omega_{n}$ of $0.014 \mathrm{rad} / \mathrm{sec}$ versus 0.9 and $4 \mathrm{rad} / \mathrm{sec}$ associated with RIFSF controller. Those dynamic parameters are found based on the conditions of controllability $\boldsymbol{C}$ and observability $\boldsymbol{O}$ matrices not being singular, i.e. Eqs. (16-17).
Table 1. A performance comparison of RIFSF controller and analytical calculations

\begin{tabular}{|l|c|c|c|c|}
\hline Category & $\boldsymbol{T}_{\boldsymbol{p}}(\mathbf{s e c})$ & $\boldsymbol{T}_{\boldsymbol{r}}(\mathbf{s e c})$ & $\boldsymbol{T}_{\boldsymbol{s}}(\mathbf{s e c})$ & P.O. \% \\
\hline $\begin{array}{l}\text { RIFSF } \\
\text { controller }\end{array}$ & 1.7206 & 0.7754 & 1.1023 & 0.285 \\
\hline $\begin{array}{l}\text { Analytical } \\
\text { calculation }\end{array}$ & 1.8018 & 0.6360 & 1.1111 & 0.255 \\
\hline
\end{tabular}

Overall, a reasonable agreement is found between the simulation results and analytical calculations. The discrepancy between RIFSF controller and analytical calculation in $\boldsymbol{T}_{\boldsymbol{r}}$ shown in Table (1) may refer to the liner approximation of analytical expression Eq. (19), particularly for $\boldsymbol{\zeta}>\mathbf{0 . 7 7}$ [6]. RIFSF controller produces so swifter and more accurate response than $F S F$ controller for spinning stabilisation satellite. A satisfactory deadbeat response is seen from RIFSF controller in terms of overshoot and steady state error. As may be noticed, the settling time is smaller than the peak time for RIFSF controller due to the deadbeat specifications met.

Table 2. A performance comparison of FSF controller and analytical calculations

\begin{tabular}{|l|l|l|l|c|}
\hline Category & $\boldsymbol{T}_{\boldsymbol{p}}(\mathbf{s e c})$ & $\boldsymbol{T}_{\boldsymbol{r}}(\mathbf{s e c})$ & $\boldsymbol{T}_{\boldsymbol{S}}(\mathbf{s e c})$ & $\begin{array}{c}\text { P.O. } \\
\%\end{array}$ \\
\hline$F S F$ controller & 477.60 & 174.10 & 319.60 & 0.285 \\
\hline $\begin{array}{l}\text { Analytical } \\
\text { calculation }\end{array}$ & 477.20 & 177.62 & 319.65 & 0.255 \\
\hline
\end{tabular}

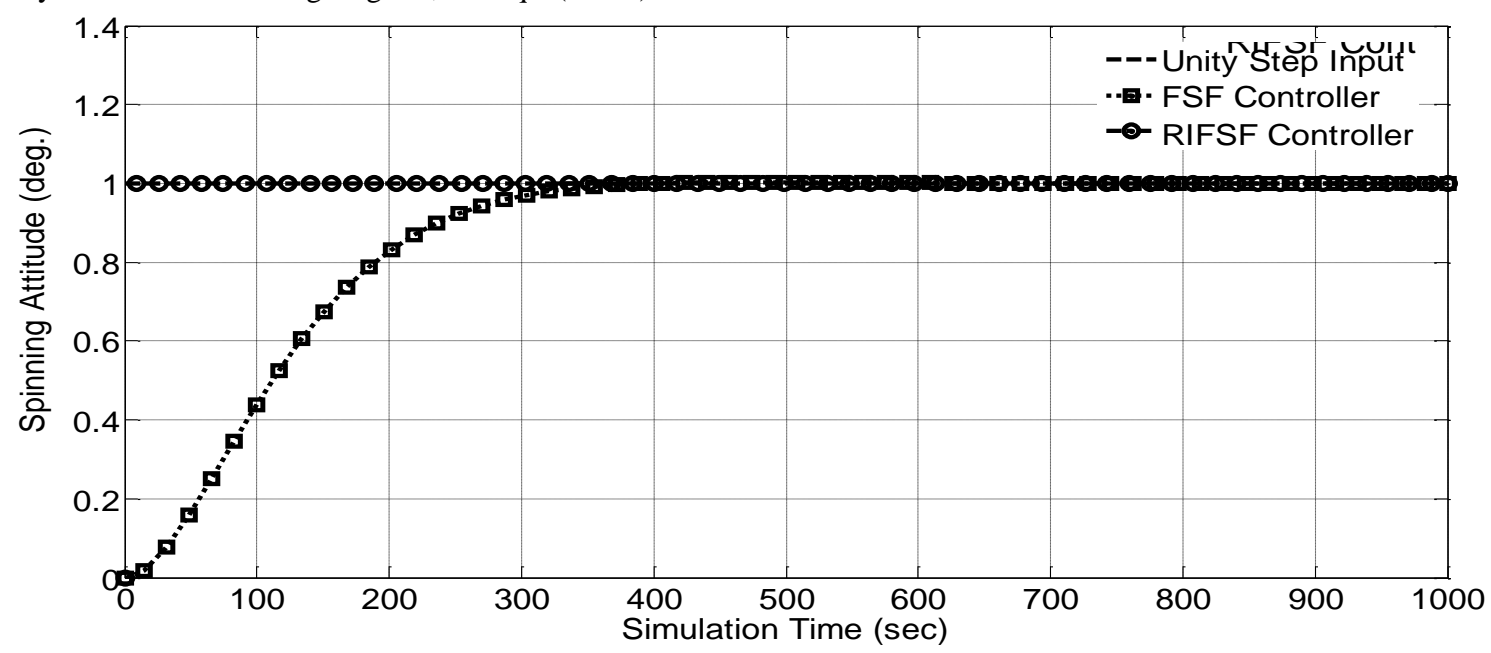

Figure 9. Deadbeat response of angular displacement attitude to unit step input command 


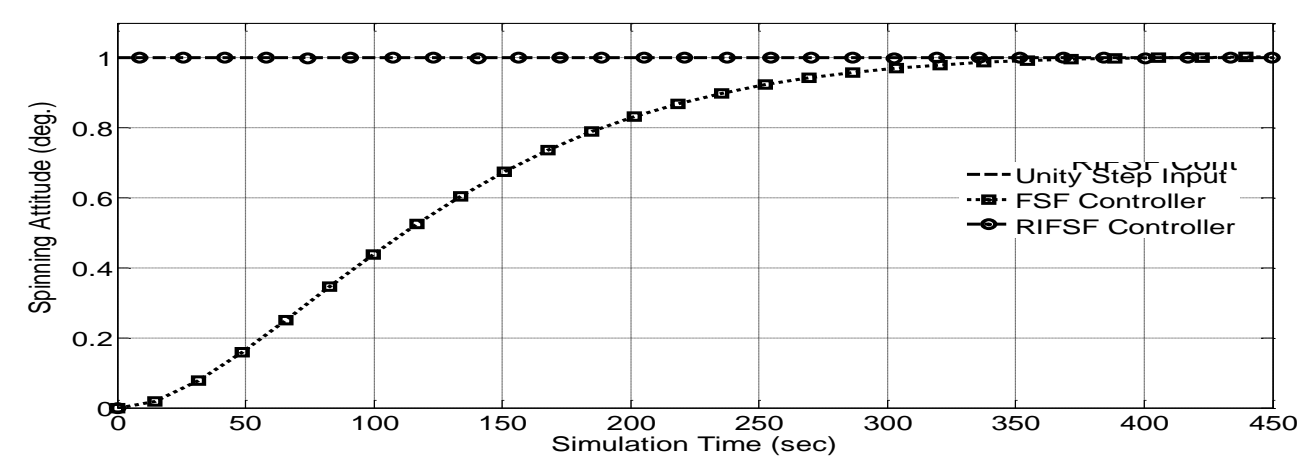

Figure 10. Zooming in view of angular displacement attitude response.

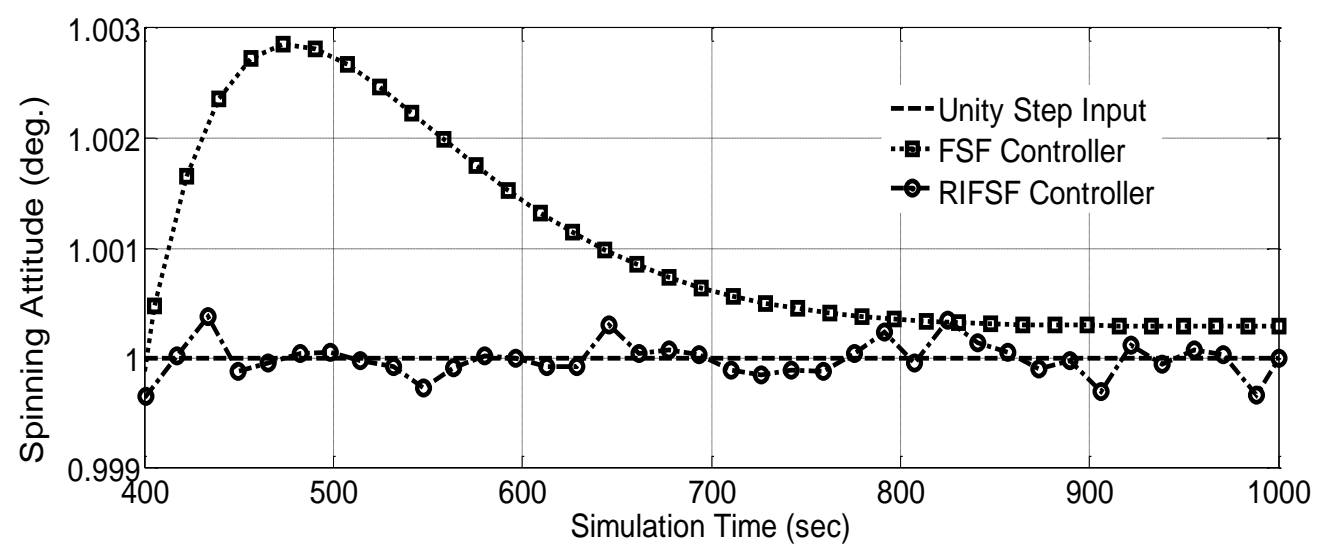

Figure 11. FSF vs RIFSF for a steady state convergence

\section{Conclusions}

This paper has been devoted to model and control spinning satellite. The satellite is intentionally chosen in an unstable mode. The aim has been to achieve a robust spin stabilization of the satellite manoeuvre. A successful implementation of two categories of $S S$ control algorithms is achieved for a satisfactory performance of spinning satellite. FSF controller is compared with RIFSF arrangement for deadbeat response to step input command. To obtain feasible values of angular velocity, ramp angular displacement command is used to excite the spinning system of a satellite. For the validation of the simulation model, the most significant results obtained from the simulation are compared with analytical results based on dominant second-order poles of 0.9-damping ratio.

Satisfactory agreements are found in terms of overshoot, rise time, settling time and steady state response. Although RIFSF meets reasonable deadbeat specifications; overshoot of $0.2 \%$, rise time of $0.65 \mathrm{sec}$, settling time of $1.2 \mathrm{sec}$ and zero steady state error, it uses impracticable large gains in comparison with FSF which shows a humble deadbeat tracking in terms of a slow response. Thus RIFSF is expansive, too accurate but reliable and fast whereas FSF is slow but cheap. However, those controller gains are not unique and they depend on the optimization relaxations. The system has very good performance and come back to its initial orientation quickly when the disturbances are zero.

Future study may be formulated to use reduced-order estimator method to confirm the selected root locations. In case of space disturbance is not excluded as they appear in a real world $S S$ control algorithms may not be robust enough to eliminate such external disturbance effects. Therefore, $S S$ controller may not be used in practical world. $\boldsymbol{H}_{\infty}$ robust attitude controller may be designed to compensate such problem.

\section{References}

[1] Gene F. Frankin, J. David Powell, and Abbas Emami-Naeini (2010). Feedback Control of Dynamic Systems. 6th ed. Pearson Prentice HallTM.

[2] Buckley, G.J. (1990). Modern Control Started with ZieglerNichols Tuning. Control Engineering, 2nd Edition, Vol. 37, No. 12, pp. 11-17.

[3] Wittenmark, B. (1979). Self -tuning PID Controllers Based on Pole Placement. Lund Inst. of Technology. Report No. TFRT7179.

[4] Kuo, Benjamin C (1994). 1930- MATLAB tools for control system analysis and design. Englewood Cliffs, NJ. Prentice Hall, 0130346462

[5] Tsiotras, P. 1996). Stabilisation and Optimality Results for the Attitude Control Problem. Journal of Guidance, Control, and Dynamics, Vol. 19, No. 4.

[6] Dorf, C.R. (1989). Modern Control Systems. 5th edition Reading, Ma. Addison- Wesley

[7] E. Finlay-Freundlich (1958). Celestial Mechanics. Pergamon press.

[8] Fortescue, M. (1996). Spacecraft Systems Engineering. McGraw Hill.

[9] Peter Fortescue. (1994). Spacecraft Systems Engineering. Wiley Publishers. 